\title{
Isotope identification capabilities using time resolved prompt gamma emission from epithermal neutrons
}

\author{
G. Festa, ${ }^{a, b, c, 1}$ L. Arcidiacono,${ }^{a}$ A. Pappalardo, ${ }^{d}$ T. Minniti, ${ }^{e}$ C. Cazzaniga, ${ }^{f}$ A. Scherillo, ${ }^{f}$ \\ C. Andreani ${ }^{a, b, e}$ and R. Senesi ${ }^{a, b, e}$ \\ a Physics Department,Università degli Studi di Roma 'Tor Vergata', \\ Via della Ricerca Scientifica 1, Italy \\ ${ }^{b}$ Museo Storico della Fisica e Centro Studi e Ricerche Enrico Fermi, \\ Piazza del Viminale 1, Italy \\ ${ }^{c}$ CNR-IC Istituto di Cristallografia, \\ Via Giovanni Amendola, 122/O, 70126, Bari, Italy \\ ${ }^{d}$ INFN Laboratori Nazionali del Sud, \\ via S.Sofia 62, 95123, Catania, Italy \\ ${ }^{e}$ CNR-IPCF Istituto per i Processi Chimico-Fisici, \\ Viale Ferdinando Stagno d'Alcontres 37, 98158, Messina, Italy \\ ${ }^{f}$ Science and Technology Facilities Council, ISIS Spallation Neutron Source, \\ Chilton-Didcot, OX11 0QX, United Kingdom \\ E-mail: giulia.festa@roma2.infn.it
}

ABSTRACT: We present a concept of integrated measurements for isotope identification which takes advantage of the time structure of spallation neutron sources for time resolved $\gamma$ spectroscopy.

Time resolved Prompt Gamma Activation Analysis (T-PGAA) consists in the measurement of gamma energy spectrum induced by the radioactive capture as a function of incident neutron Time Of Flight (TOF), directly related with the energy of incident neutrons. The potential of the proposed concept was explored on INES (Italian Neutron Experimental Station) at the ISIS spallation neutron source (U.K.). Through this new technique we show an increase in the sensitivity to specific elements of archaeometric relevance, through incident neutron energy selection in prompt $\gamma$ spectra for multicomponent samples. Results on a standard bronze sample are presented.

KeYwords: Instrumentation and methods for time-of-flight (TOF) spectroscopy; Inspection with neutrons; Instrumentation for neutron sources; Gamma detectors (scintillators, CZT, HPG, HgI etc)

${ }^{1}$ Corresponding author. 


\section{Contents}

1 Introduction 1

2 Experiment 2

3 Results and discussion $\quad 2$

4 Conclusions $\quad 8$

\section{Introduction}

The strength of neutron imaging techniques lies in the unique properties of neutron-matter interaction, such as large penetration depth into bulk materials, sensitivity to light atoms and high contrast between adjacent elements in the periodic table [1]. The advent of advanced pulsed neutron sources has made available intense fluxes of epithermal neutrons, with energies from above $0.5 \mathrm{eV}$ up to the $\sim 1000 \mathrm{eV}$ range [2]. State of the art technologies available for neutron-based methods, using cold, thermal and epithermal-fast neutron beams, are currently being applied in various fields such as engineering, materials science and Cultural Heritage at several neutron beam facilities throughout the world [1].

Two non-destructive neutron techniques using the prompt $\gamma$ emission following neutron interaction have been mostly employed up to now for elemental characterisation: Prompt Gamma Activation Analysis (PGAA) and Neutron Resonance Capture Analysis (NRCA). Both techniques exploit the $(n, \gamma)$ process in a complementary manner. NRCA [3] exploits the pulsed nature of a spallation source. Quantitative information about elemental and isotope compositions is obtained through Time Of Flight (TOF) measurements. The technique makes use of $\gamma$-detectors to detect the $\gamma$-ray emission following a resonant neutron capture event in the sample, and records the related TOF. Different elements, and their relative abundances, are therefore distinguished according to element-specific characteristics of TOF spectra, i.e. according to the relative intensity of TOF peaks. In PGAA [4], $\gamma$ spectroscopy is carried out with high resolution $\gamma$-ray spectrometers. The technique generally employs thermal and cold neutrons and gamma energy spectra are recorded [5-7] without any TOF information. PGAA can be therefore successfully applied on continuous neutron sources.

Dual TOF-photon energy resolved analysis goes beyond the current state of the art of both PGAA and NRCA, therefore enhancing the capabilities of both techniques and providing simultaneous element/isotope sensitivity currently not available. In this paper we present initial results on a standard bronze sample measured through this new technique. 


\section{Experiment}

Measurements were performed at the INES beamline, a general purpose diffractometer mainly devoted to materials characterization, cultural heritage studies and equipment tests, operating at the ISIS pulsed spallation neutron source (U.K.). The INES beam line is placed on a decoupled-Gd poisoned water moderator at $T=295 \mathrm{~K}$ that provides a neutron spectrum peaked at $E \approx 30 \mathrm{meV}$ and a $1 / E$ tail in the epithermal-fast neutron region. The primary flight path, from the moderator to the sample position is $L \approx 22.8 \mathrm{~m}$; samples arelocated inside an aluminium sample tank. The incident neutron flux is in the order of $1 \times 10^{7} \mathrm{n} / \mathrm{cm}^{2} / \mathrm{s}$ [8]. Measurements of the $\gamma$-ray spectra on INES were performed using an ORTEC-GMX15 HPGe detector with quoted efficiency varying between $25 \%$ at $60 \mathrm{keV}$ and $3.7 \%$ at $1332 \mathrm{keV}$. The detector was placed $1 \mathrm{~m}$ from the sample position at 90 degrees with respect to the neutron incident beam direction due to sample tank constrains. The HpGe output signal was sent to a channel of a DT5724-4 Channel CAEN digitizer. An attenuator was used to adjust the signal intensity to the input digitiser dynamic range. A copy of the spallation proton pulse signal is available on the INES Data Acquisition Electronics, and was used to synchronize the acquisition with the neutron pulses. This signal, referred to as $T_{0}$ from the ISIS source, is a TTL signal that corresponds to the time at which the high energy proton beam hits the target and neutrons are produced for each pulse. The neutron pulses are produced at a fixed frequency of $50 \mathrm{~Hz}$. The $T_{0}$ signal was fed to a second channel of the digitizer to obtain the time synchronization.

A recorded event is defined by a signal above a defined threshold. Since the digitizer records a time stamp for each event with $10 \mathrm{~ns}$ accuracy, the TOF of each event is computed as the difference of the two time stamps. A trapezoidal filter algorithm is used by the digitizer's FPGA to calculate the signal Pulse Height, which is proportional to the energy deposited in the HPGe by the $\gamma$-ray. The result of this process is that a bi-parametric acquisition (TOF-photon energy) is possible. In fact, for each signal both the Pulse Height (i.e. the deposited energy) and the neutron TOF are recorded and written to a file. Data analysis was carried out through the ROOT software environment $[9,10]$, using custom developed macros. The macros produce a two-dimensional matrix containing counts as function of: 1) neutron time-of flight that is strictly related to the incident neutron energy; 2) deposited $\gamma$-ray energy in the HPGe.

The gamma energy range obtained from the two-dimensional matrix is $10 \mathrm{keV}<E_{\gamma}<8 \mathrm{MeV}$, while the neutron time of flight range in the two-dimensional matrix is $0.1 \mathrm{eV}<E_{n}<68 \mathrm{eV}$. The data can be represented either as: 1) a prompt gamma spectrum as a function of the incident neutron energy, the latter defined through the neutron time of flight from the pulsed source to the sample; 2) an NRCA spectrum as a function of the gamma energy. The sample used in the present measurements is a certificated leaded bronze standard sample from the MBH Analytical LTD (code $32 \mathrm{X}$ LB10 E), a disk with a diameter of $40 \mathrm{~mm}$ and thickness of $4 \mathrm{~mm}$. The composition and element percentage by weight of the sample are reported in table 1 .

\section{Results and discussion}

Figure 1 reports the count intensity bi-parametric map of $\gamma$-ray energy vs. TOF spectrum of the bronze sample. From the inspection of the intensity map it is possible to follow the TOF evolution of the individual $\gamma$-ray lines such as for example the $511 \mathrm{keV}$ annihilation and $478 \mathrm{keV}$ boron capture 
Table 1. Element composition of the standard bronze sample: $\mathrm{Cu}(77.98 \pm 0.10), \mathrm{Sn}(8.16 \pm 0.09), \mathrm{Pb}$ $(11.74 \pm 0.07), \mathrm{Zn}(0.566 \pm 0.09), \mathrm{Fe}(0.013 \pm 0.001), \mathrm{Ni}(0.687 \pm 0.010), \mathrm{Al}(0.0005), \mathrm{Si}(0.001), \mathrm{Co}$ $(0.0456 \pm 0.0014), \mathrm{As}(0.151 \pm 0.003), \mathrm{Mn}(0.0004 \pm 0.0001), \mathrm{Bi}(0.0608 \pm 0.0010), \mathrm{Sb}(0.591 \pm 0.008), \mathrm{Ag}$ $(0.042 \pm 0.002), \mathrm{P}(0.001), \mathrm{S}(0.0116 \pm 0.0011)$.

\begin{tabular}{|l|l|l|l|l|l|l|l|l|}
\hline $\begin{array}{l}\text { 32X LB10 E } \\
\text { standard } \\
\text { bronze }\end{array}$ & $\mathrm{Cu}$ & $\mathrm{Sn}$ & $\mathrm{Pb}$ & $\mathrm{Zn}$ & $\mathrm{Fe}$ & $\mathrm{Ni}$ & $\mathrm{Al}$ & $\mathrm{Si}$ \\
\hline $\begin{array}{l}\text { Weight compo- } \\
\text { sition (\%) }\end{array}$ & 77.98 & 8.16 & 11.74 & 0.566 & 0.013 & 0.687 & 0.0005 & 0.001 \\
\hline $\begin{array}{l}32 \mathrm{X} \text { LB10 E } \\
\text { standard } \\
\text { bronze }\end{array}$ & $\mathrm{Co}$ & $\mathrm{As}$ & $\mathrm{Mn}$ & $\mathrm{Bi}$ & $\mathrm{Sb}$ & $\mathrm{Ag}$ & $\mathrm{P}$ & $\mathrm{S}$ \\
\hline $\begin{array}{l}\text { Weight compo- } \\
\text { sition (\%) }\end{array}$ & 0.0456 & 0.151 & 0.0004 & 0.0608 & 0.591 & 0.042 & 0.001 & 0.0116 \\
\hline
\end{tabular}

across the resonance and out of resonance region. The evolution of the intensities along the vertical axis ( $\gamma$-energy) allows to identify the gamma spectral contributions to resonance and off-resonance intensities.

Cuts along the vertical axis provide time of flight-resolved PGAA spectra, while cuts along the horizontal axis provide gamma energy-resolved NRCA spectra. The width of the cuts can be

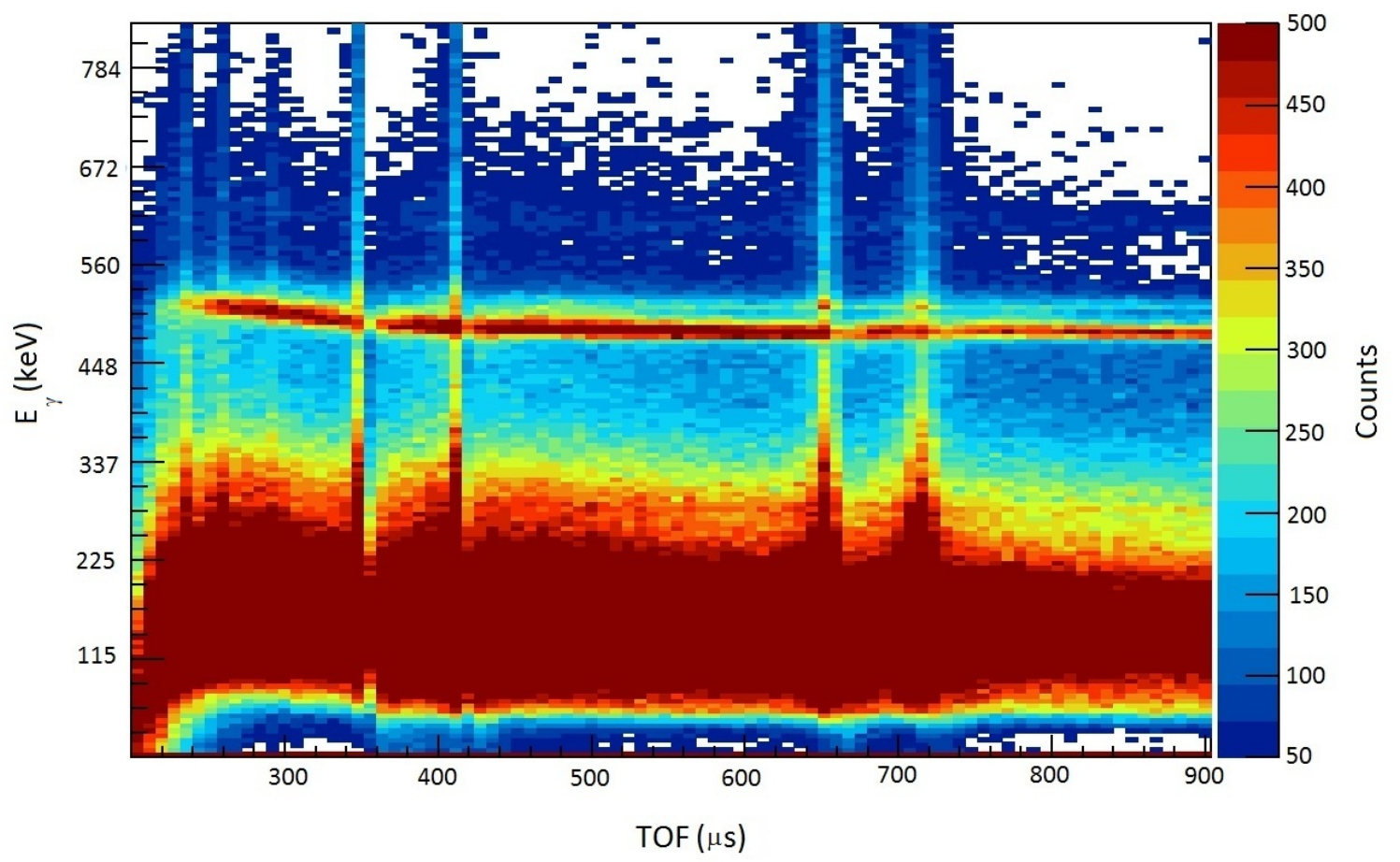

Figure 1. Count intensity map of the bi-parametric ( $\gamma$-ray energy vs. time-of-flight) spectrum measured with the HPGe detector of the bronze standard sample (MBH Analytical LTD 32X LB10). 


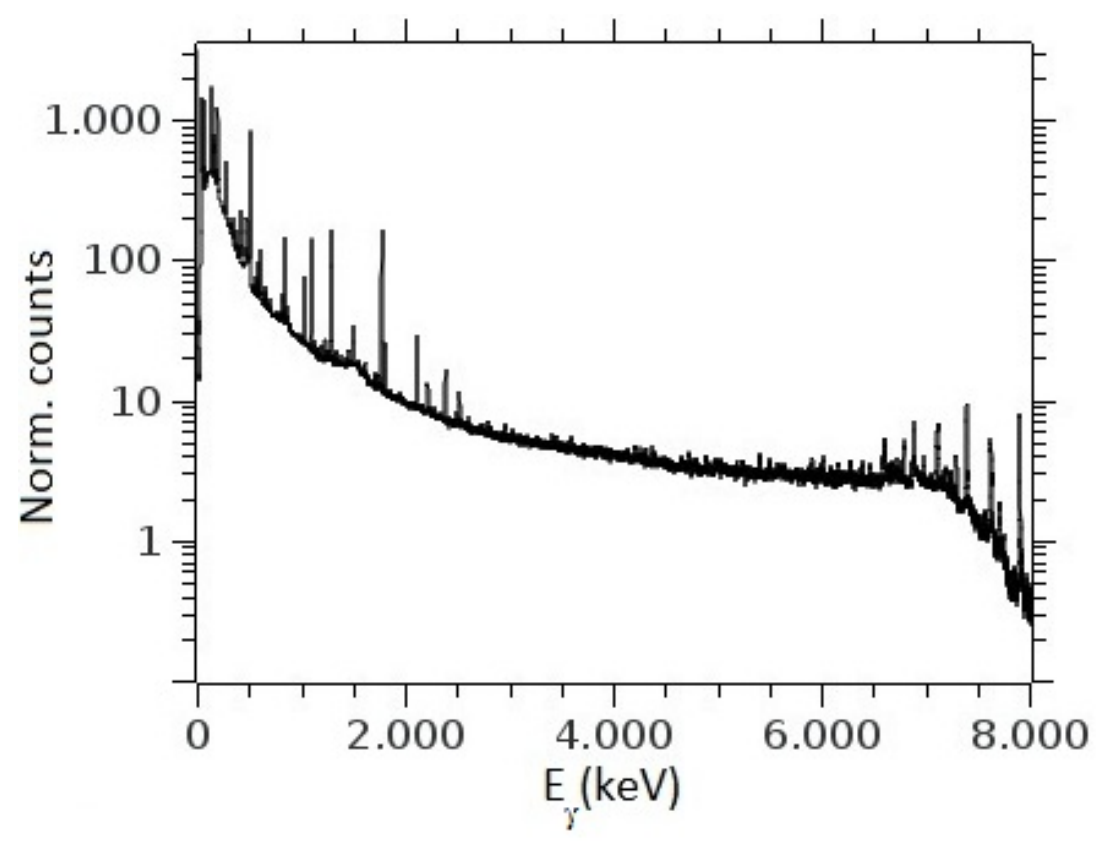

Figure 2. PGAA spectrum of the bronze standard sample (MBH Analytical LTD 32X LB10) using neutrons in the range $2 \mathrm{eV}<\mathrm{E}_{n}<68 \mathrm{eV}$. Counts were normalised to the time acquisition.

adjusted and widened to increase the sensitivity to the isotopic contents due to the isotopic variation of the $(n, \gamma)$ cross sections as a function of neutron energy and of the isotope-dependent $\gamma$ cascades. For example, it is possible to derive a PGAA from electron Volt neutrons, as compared to a PGAA from thermal neutrons, or a photon energy resolved NRCA to reduce or enhance particular $\gamma$-ray lines associated to a specific isotope.

As an example, figure 2 reports a PGAA spectrum from $\mathrm{eV}$ neutrons: from the bronze sample obtained by bi-parametric acquisition integrated all over the TOF range from 200 to $2000 \mu \mathrm{s}$, and corresponding to an incident neutron energy between $2 \mathrm{eV}$ and $68 \mathrm{eV}$ approximately. The $\gamma$ spectrum extends approximately from $10 \mathrm{keV}$ to $8000 \mathrm{keV}$. It is composed of individual $\gamma$-ray lines from the sample and $\gamma$-ray lines from the instrument background. The latter is due to the interaction of the incident and transmitted neutrons with the beamline components and has been characterised previously [11]. The overall spectrum is composed of more than $70 \gamma$-ray lines and the continuous Compton contribution [4].

The sample spectrum contains background contribution arising from both prompt and activation spectra which often shows peaks overlapping with the samples' $\gamma$-ray lines. To this end, the biparametric acquisition can be integrated in the photon energy range from $10 \mathrm{keV}$ to $8000 \mathrm{keV}$ so as to provide a Neutron Resonance Capture Analysis spectrum in the $\mathrm{eV}$ range. This projection allows to enhance the signal from elements in the sample having neutron resonances in the $\mathrm{eV}$ range.

Figure 3 reports the NRCA spectrum of the bronze sample obtained by integrating the biparametric spectrum in the above gamma energy range. The spectrum is reported in the time-offlight range $200 \mu \mathrm{s}$ to $2000 \mu$ s corresponding to a neutron energy of $68 \mathrm{eV}$ down to $2 \mathrm{eV}$. Several resonances can be observed, namely three rather evident resonances from silver at $294 \mu \mathrm{s}(31 \mathrm{eV})$, $412 \mu \mathrm{s}(16 \mathrm{eV})$ and $717 \mu \mathrm{s}(5.3 \mathrm{eV})$, one resonance from tin at $257 \mu \mathrm{s}(41 \mathrm{eV})$ and two resonances 


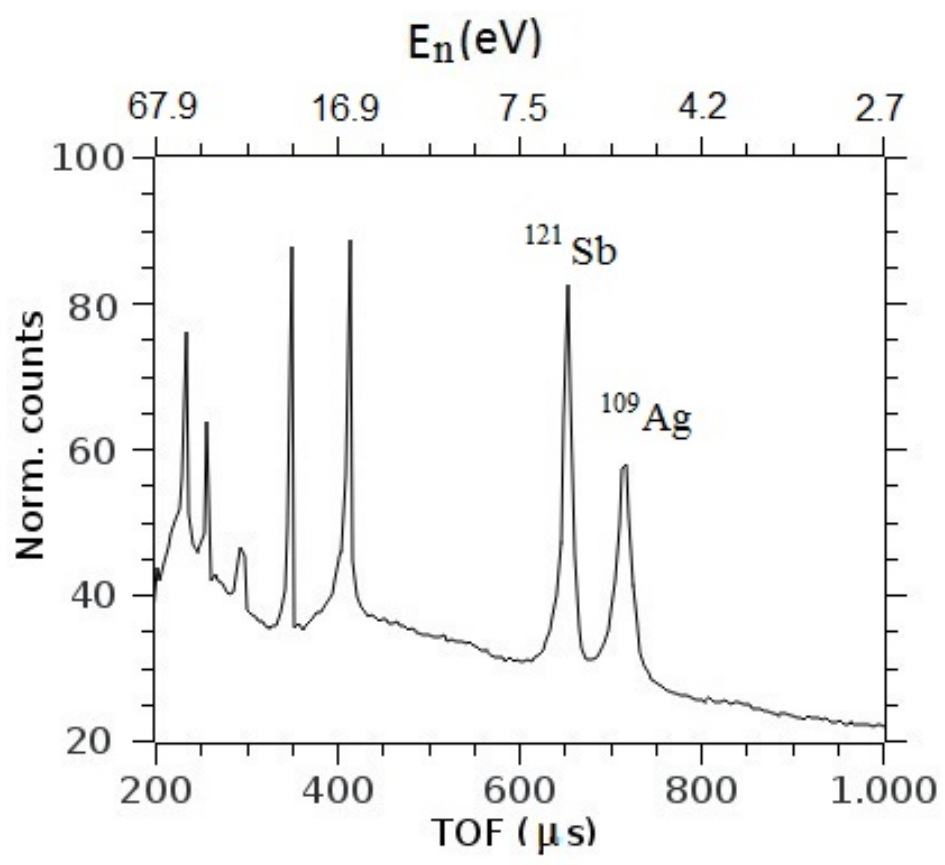

Figure 3. Time-of-flight NRCA spectrum from of the bronze standard sample (MBH Analytical LTD 32X LB10). Counts were normalised to the time acquisition.

from antimony at $349 \mu \mathrm{s}(22.3 \mathrm{eV})$ and $653 \mu \mathrm{s}(6.4 \mathrm{eV})$ [3]. Some of other elements in the sample witch have resonances above $68 \mathrm{eV}$, such as copper and lead, were not detected because the instrumental set-up introduced a low time of flight cut-off at $200 \mu \mathrm{s}$.

Figure 2 and figure 3 show that PGAA and NRCA provide complementary information on the sample element composition. These results show the potential for the use of $(n, \gamma)$ techniques using epithermal neutrons, with special regards to neutron energy-resolved PGAA. The latter is indeed routinely carried out using thermal or cold neutrons [4] and figure 2 show that the feasibility of PGAA with eV neutrons can be assessed. However the joint use of PGAA and NRCA results in the loss of part of the information contained in the physical process of radiative resonance absorption does not allow unambiguous identification to the neutron induced photon emission. In particular: in the PGAA spectrum which $\gamma$-ray lines are associated to a specific resonance? And in the NRCA spectrum which $\gamma$-ray lines contribute to the observed resonances? These questions can only be answered if the time of flight and gamma energy spectra are acquired in event mode that is in a fully bi-parametric acquisition. An example of the potential of the present technique is shown in figure 4 . PGAA can be acquired as a function of the neutron TOF (energy), allowing an enhancement or a decrease of specific $\gamma$ lines associated to specific isotopic species in the sample, which depend on the differences in the $(n, \gamma)$ cross sections as a function of energy of the isotopic species. Figure 4 , upper panel, show that by selecting the neutron energy in the range $0.11 \mathrm{eV} \leq E_{n} \leq 0.17 \mathrm{eV}$, the PGAA signal at $164 \mathrm{keV}$ associated to ${ }^{107} \mathrm{Ag}$ is considerably enhanced with respect to the neutron energy range $0.30 \mathrm{eV} \leq E_{n} \leq 0.67 \mathrm{eV}$. This is due to the different energy variation of the ${ }^{107} \mathrm{Ag}$ 

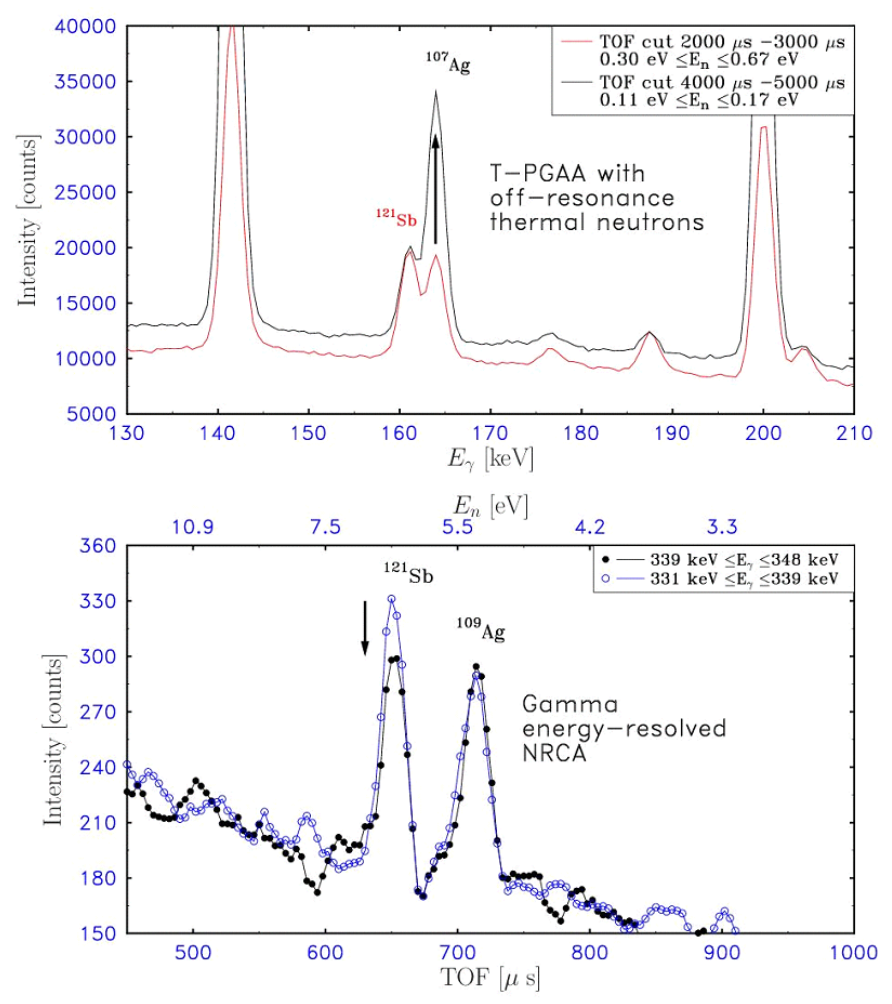

Figure 4. Upper panel: T-PGAA with neutron energies selected in the range $0.11 \mathrm{ev} \leq E_{n} \leq 0.17 \mathrm{ev}$ (black continuous line), and T-PGAA with neutron energies selected in the range $0.30 \mathrm{ev} \leq E_{n} \leq 0.67 \mathrm{ev}$ (red continuous line). The gamma lines from ${ }^{107} \mathrm{Ag}$ at $164 \mathrm{keV}$ and from ${ }^{121} \mathrm{Sb}$ at $161 \mathrm{keV}$ are indicated in the plot; the black arrow shows the relative increase of the $164 \mathrm{keV}$ line obtained by selective neutron TOF and energy cuts. Lower panel: gamma energy-resolved NRCA spectra using the gamma energy selection $331 \mathrm{keV}$ $\leq E_{\gamma} \leq 339 \mathrm{keV}$ (blue open circles), and $339 \mathrm{keV} \leq E_{\gamma} \leq 348 \mathrm{keV}$ (black filled circles). The NRCA peaks from ${ }^{121} \mathrm{Sb}$ and ${ }^{109} \mathrm{Ag}$ are indicated in the plot; the black arrow shows the relative decrease of the ${ }^{121} \mathrm{Sb}$ resonance peak obtained from the gamma energy selection $339 \mathrm{keV} \leq E_{\gamma} \leq 348 \mathrm{keV}$.

$(n, \gamma)$ cross section as compared to ${ }^{121} \mathrm{Sb}$, even outside of the resonance regions at $\mathrm{eV}$ energies. This opens the possibility to develop tunable isotopic contrast using T-PGAA.

Figure 4, lower panel, reports an example of $\gamma$ energy-resolved NRCA spectra using horizontal ( $\gamma$-energy) cuts in the biparametric matrix: the relative height of the resonances at $5.2 \mathrm{eV}$ from ${ }^{109} \mathrm{Ag}$ and at $6.2 \mathrm{eV}$ from ${ }^{121} \mathrm{Sb}$ can be varied according to the selected $\gamma$ energy window. In particular, specific $\gamma$ lines contributing to the NRCA peak from ${ }^{121} \mathrm{Sb}$ can be selectively included or excluded from the NRCA spectrum. Neutron-gamma databases, such as CAPGAM [12], can be used in addition to the analysis of the PGAA spectrum as a guide to the selection or exclusion of the gamma lines. The gamma energy range reported as blue open circles in figure 4 corresponds to $331 \mathrm{keV}$ $\leq E_{\gamma} \leqslant 339 \mathrm{keV}$. In this range two intense $(n, \gamma)$ lines from ${ }^{121} \mathrm{Sb}$ contribute to the NRCA peak: 1$)$ $331.3 \mathrm{keV}$ with $1.5 \%$ relative intensity with respect to the strongest transition at $61.4 \mathrm{keV}$ [12]; 2) $332.3 \mathrm{keV}$ with $8.3 \%$ relative intensity with respect to the strongest transition at $61.4 \mathrm{keV}$ [12]. As far as the ${ }^{109} \mathrm{Ag}$ resonance is concerned, five low intensity $(n, \gamma)$ lines $(<0.05 \%$ relative intensity 


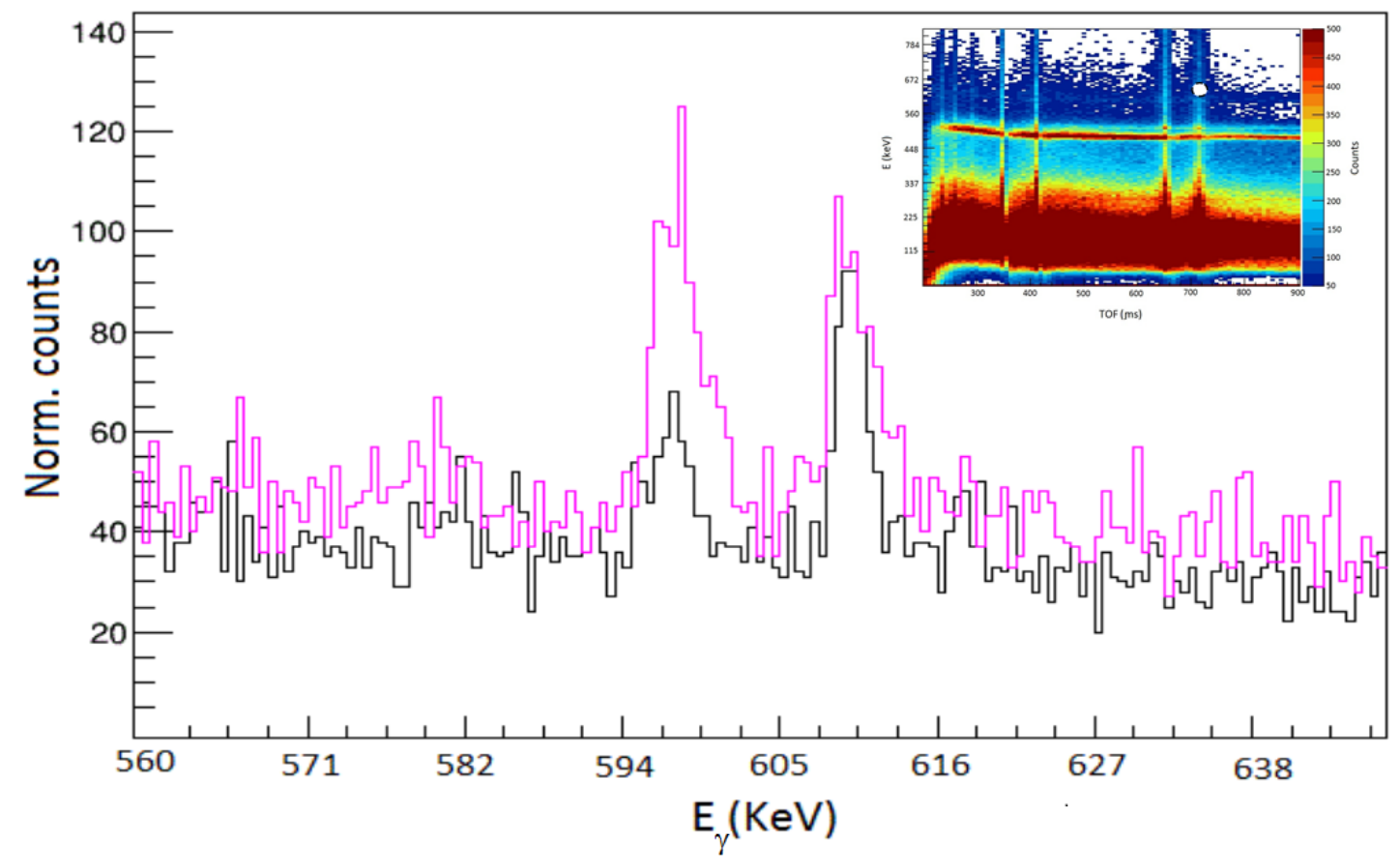

Figure 5. T-PGAA spectra in the energy range $560 \mathrm{keV} \leq E_{\gamma} \leq 640 \mathrm{keV}$ with a time of flight cut corresponding to the ${ }^{109} \mathrm{Ag}$ resonance at $5.2 \mathrm{eV}$ (from 690 to $720 \mu \mathrm{s}$ ) reported in pink, and off-resonance condition (from 1800 to $1830 \mu \mathrm{s}$ ) reported in black. The counts for the two spectra are normalised to have the same intensities at $560 \mathrm{keV}$. The inset reports the biparametric TOF/gamma energy cut (white filled circle) used to obtain the T-PGAA spectra.

with respect to the strongest transition at $198.7 \mathrm{keV}$ [12]) contribute to the NRCA peak at $5.2 \mathrm{eV}$ from ${ }^{109} \mathrm{Ag}$. If the $\gamma$ energy range is changed to $339 \mathrm{keV} \leq E_{\gamma} \leq 348 \mathrm{keV}$, only a single $(n, \gamma)$ line $(<0.85$ $\%$ relative intensity with respect to the strongest transition at $198.7 \mathrm{keV}$ [12]) from ${ }^{121} \mathrm{Sb}$ contribute to the resonance peak, and the intensity of the $6.2 \mathrm{eV}$ resonance is reduced. On the other hand, as for the previous case, five low intensity $(n, \gamma)$ lines $(<0.05 \%$ relative intensity with respect to the strongest transition at $198.7 \mathrm{keV}$ [12]) contribute to the NRCA peak at $5.2 \mathrm{eV}$ from ${ }^{109} \mathrm{Ag}$, with no intensity reduction. The NRCA spectrum corresponding to this second $\gamma$ range selection is reported as black filled circles in figure 4 . The figure shows that the NRCA peak at $6.2 \mathrm{eV}$ from ${ }^{121} \mathrm{Sb}$ is selectively reduced providing a complementary adjustable isotopic contrast between the ${ }^{109} \mathrm{Ag}$ and ${ }^{121} \mathrm{Sb}$ signals.

As a further example of increased sensitivity, in figure 5 we report a cut in the TOF range $(690 \mu \mathrm{s} \leqslant \mathrm{TOF} \leqslant 720 \mu \mathrm{s})$ corresponding to the $5.2 \mathrm{eV}{ }^{109} \mathrm{Ag}$ resonance, compared with a cut in the out of resonance TOF range $(1800 \mu \mathrm{s} \leq \mathrm{TOF} \leq 1830 \mu \mathrm{s})$. The spectrum is reported in the $\gamma$ energy range $560 \mathrm{keV} \leq E_{\gamma} \leq 640 \mathrm{keV}$. The spectrum shows a gamma energy doublet with energies at approximately $597 \mathrm{keV}$ from ${ }^{109} \mathrm{Ag}$ and $611 \mathrm{keV}$ from ${ }^{123} \mathrm{Sb}$ [12].

The ${ }^{109} \mathrm{Ag}$ T-PGAA peak, increases in relative intensity, as compared to the ${ }^{123} \mathrm{Sb}$ T-PGAA peak when the incident neutron TOF/energy cut corresponds to the silver resonance. This allows an univocal attribution of the peak at $597 \mathrm{keV}$ to ${ }^{109} \mathrm{Ag}$. The examples outlined in this section outline the potential for tunable isotopic contrast and selectivity using T-PGAA and gamma energy-resolved NRCA. Further development are envisaged through the use of multiple selective cuts in both TOF 
and $\gamma$ energy, and are the subject of planned analysis and experiments. We point out that, by the use of pencil beam geometry, two dimensional scanning capabilities with tunable isotopic sensitivity may be achieved and will pursued in the next future.

\section{Conclusions}

The work presents a new concept of integrated methodology for elemental and isotope identification that takes advantage of the time structure of a spallation neutron source for time resolved $\gamma$ spectroscopy. Effectiveness of the technique is demonstrated through the analysis of a standard bronze sample measured through a bi-parametric time of flight and $\gamma$-acquisition. Time resolved Prompt Gamma Activation Analysis consists in the $\gamma$-ray energy measurement induced by the radioactive capture as a function of incident neutron time of flight, directly related with the energy of incident neutron. The potential of the proposed concept was explored on INES (Italian Neutron Experimental Station) at the ISIS spallation neutron source (U.K.). This dual Time of Flight-photon energy resolved analysis, acquired in event mode, goes beyond the current state of the art. It has the potential to enhance the knowledge of the $(n, \gamma)$ process in materials and the capabilities of both the NRCA and PGAA techniques, providing a simultaneous element and isotope sensitivity not currently available. Results show that selecting neutron energies through time-windows acquisition centred around resonances' energies of the minority elements present in the sample increases the sensitivity, broadening the scope and capabilities of electron Volt neutron instrumentation [13].

Through incident neutron and $\gamma$-ray collimation, the new technique can be used to perform elemental and isotope mapping of the irradiated sample with higher element sensitivity than currently available.

\section{Acknowledgments}

This work was supported within the CNR-STFC Agreement 2014-2020 concerning collaboration in scientific research at the ISIS spallation neutron source.

\section{References}

[1] H.Z. Bilheux, R. McGreevy and I.S. Anderson eds., Neutron Imaging and Applications, Springer (2009), doi:10.1007/978-0-387-78693-3.

[2] C. Windsor, Pulsed neutron scattering, Taylor \& Francis (1981).

[3] H. Postma and P. Schillebeeckx, Neutron Resonance Capture and Transmission Analysis, in R.A. Meyers ed., Encyclopedia of Analytical Chemistry, Wiley, New York (2009), doi:10.1002/9780470027318.a9070.

[4] G.L. Molnar, Handbook of Prompt Gamma Activation Analysis with neutron beams, Kluwer Academic Publishers (2004).

[5] Ancient Charm collaboration, T. Belgya et al., First elemental imaging experiments on a combined PGAI and NT setup at the Budapest Research Reactor, J. Radioanal. Nucl. Ch. 278 (2008) 751.

[6] Ancient Charm collaboration, T. Belgya et al., A new PGAI-NT setup at the NIPS facility of the Budapest Research Reactor, J. RadioanalNucl. Ch. 278 (2008) 713. 
[7] Ancient Charm collaboration, G. Festa et al., A nondestructive stratigraphic and radiographic neutron study of Lorenzo Ghiberti's reliefs from paradise and north doors of Florence baptistery, J. Appl. Phys. 106 (2009) 074909.

[8] S Imberti et al., Neutron diffractometer INES for quantitative phase analysis of archaeological objects, Meas. Sci. Technol. 19 (2008) 034003.

[9] R. Brun and F. Rademakers, Root - an object oriented data analysis framework, in Proceedings AIHENP'96 Workshop, Lausanne, 1996, http://root.cern.ch/.

[10] R. Brun and F. Rademakers, ROOT: An object oriented data analysis framework, Nucl. Instrum. Meth. A 389 (1997) 81.

[11] A. Miceli et al., Measurements of gamma-ray background spectra at spallation neutron source beamlines, J. Anal. Atom. Spectrom. 29 (2014) 1897.

[12] http://www.nndc.bnl.gov/capgam/index.html.

[13] A. Pietropaolo and R. Senesi, Electron Volt neutronspectrometers, Phys. Rep. 508 (2011) 45. 